\title{
On the performance of cognitive full-duplex relaying under spectrum sharing constraints
}

\author{
Samuel B. Mafra ${ }^{1 *}{ }^{*}$, Hirley Alves ${ }^{1,2+}$, Daniel B. Costa $^{3}$, Richard D. Souza ${ }^{1 \dagger}$, Evelio M. G. Fernandez ${ }^{4}$ \\ and Matti Latva-aho ${ }^{2}$
}

\begin{abstract}
In this paper, a new full-duplex (FD) relaying scheme for a cooperative cognitive underlay network is proposed. The secondary network is composed of one secondary transmitter, one full-duplex secondary relay, and one secondary destination. The relay employs the selective-decode-and-forward (SDF) protocol. The secondary destination jointly decodes the signals from the secondary transmitter and the FD secondary relay so that the direct link can be seen as useful information rather than interference. The analysis includes the effect of the interference from the primary transmitter and the self-interference at the relay. Under equal power allocation strategy, closed-form expressions for the outage probability are derived for the proposed FD cooperative cognitive scheme, and the feasibility of FD relaying under cognitive constraints is shown. Our results also reveal that the proposed full-duplex joint-decoding (FDJD) cognitive network considerably outperforms the known full-duplex dual-hop (FDDH) scheme. Moreover, we propose an optimal power allocation (OPA) scheme. On the basis of the signal-to-interference-plus noise of the secondary network, the OPA strategy can choose between two modes of operation, cooperation between source and relay or source transmission only. Our results show that the FDJD scheme under the proposed OPA policy presents the best performance among all schemes investigated in this paper.
\end{abstract}

Keywords: Cooperative cognitive networks; Full-duplex relaying; Joint-decoding; Self-interference; Spectrum sharing

\section{Introduction}

With the objective of obtaining a more efficient utilization of the radio spectrum, in a cognitive radio network, the unlicensed users (also referred to as secondary users) may communicate using frequencies of the licensed spectrum [1-4]. The most practical cognitive radio protocols are interweave and underlay [1]. In the interweave protocol, the unlicensed users monitor the radio spectrum and communicate over spectrum holes without causing interference to the licensed users (primary users). Differently from the interweave protocol, in the underlay protocol, the secondary network may transmit concurrently with the primary network as long as the primary communication is not compromised. In this scenario, a maximum allowable interference level at the primary receiver is defined so that the secondary users (SUs) must take into account this threshold during the transmission in order

\footnotetext{
*Correspondence: mafrasamuel@gmail.com

${ }^{\dagger}$ Equal contributor

1 Federal University of Technology_Paraná (UTFPR), 80230-901 Curitiba, Brazil Full list of author information is available at the end of the article
}

to adjust their transmit powers. This allows a more efficient use of the frequency spectrum since the interference is limited at the primary receiver [1-4].

Recently, cooperative communications emerged as an alternative technique to boost the performance of communication systems $[5,6]$. The idea behind this strategy is to make use of one or more nodes, known as relay(s), to help the communication between source and destination. The result is a virtual antenna array formed by single antenna devices. Thus, through cooperation, the same benefits obtained in multiple-input multiple-output (MIMO) systems can be achieved. The relay behavior is governed by cooperative protocols which can operate either on half-duplex (HD) or on full-duplex (FD) modes, using parallel or repetition coding, selective or incremental strategies, etc. [5, 7-9]. Specifically, in HD mode, the relay transmits and receives in orthogonal channels, whereas in FD mode, the transmission and reception are performed at the same time and at the same frequency band. Owing to this fact, HD relays require the use of additional system resources, while FD relays arise as a viable

\section{量 Springer}

(C) 2015 Mafra et al. This is an Open Access article distributed under the terms of the Creative Commons Attribution License (http://creativecommons.org/licenses/by/4.0), which permits unrestricted use, distribution, and reproduction in any medium, provided the original work is properly credited. 
option to alleviate this problem. However, although ideal FD relaying can achieve higher capacity than HD relaying [8], its use introduces self-interference since perfect isolation between transmitted and received signals is hard to be guaranteed [8-12].

Nevertheless, the works [9-17] showed that FD is feasible even in the presence of high self-interference levels. Even though sophisticated signal processing techniques are employed at the FD node, it is unfortunate that the self-interference cannot be completely mitigated $[10,11]$. Experimental results in [10] showed that with advanced signal processing together with antenna isolation techniques, self-interference can be considerably reduced but not to the noise level. Thus, residual interference still remains, and it can be modeled as a fading channel as investigated in [9, 11-17]. For instance, in [13], it is shown that FD relaying is feasible even if the relay experiences strong self-interference, while enhancing capacity when compared with HD relaying. In $[14,15]$, the authors compare the performance of selective and incremental cooperative HD protocols to FD cooperative protocols. The authors show that FD operation can considerably increase performance in terms of outage probability as well as throughput even in the presence of strong selfinterference. More general fading settings such as Rice and Nakagami-m fading are investigated in $[16,17]$. The common conclusions among [9-17] are that FD operation is feasible and that overall performance can be considerably enhanced with respect to HD relaying.

\subsection{Cooperative cognitive networks}

Motivated by the great benefits acquired through cognitive radio and cooperative techniques, several works analyzed the performance of cooperative cognitive networks under spectrum sharing constraints, as for instance [18-29]. Nevertheless, a common characteristic of these works is that all proposed schemes operate under HD mode with exception of [29]. For instance, [18] assumes an underlaid cooperative secondary network in a multinode primary network. The authors consider that the secondary user adjusts its transmit power according to maximal interference link; however, neglecting the direct link between secondary source and destination. In [19], the authors assume that the dual-hop cooperative secondary network suffers with the primary interference and draw a discussion on how it impacts on the secondary performance.

Further, in [20], the outage probability of a dual-hop cognitive relay network under general fading setting is analyzed. Thus, assuming two different strategies to determine the transmit power of the secondary network, the authors show that the diversity order is dominated by the minimum fading severity between the two hops of the secondary network. A similar setting was investigated in [21], where the authors account for the direct link at the secondary cooperative network and show that the diversity order is solely dependent on the fading severity parameters of the secondary network. In [22], the authors ignore the direct link but include the interference of the primary network in their analysis of outage probability showing the impact on performance because of the interference from the primary network. The authors in [23] exploit the primary network retransmissions in order to increase performance of the underlaid network. The results show that cooperation at the underlaid layer considerably increases performance without harming the performance of the primary network.

In [24], the performance of a multi-relay secondary network is evaluated revealing that the diversity gain is defined by the number of available relays. Correspondingly, in [25], outage probability and ergodic capacity are evaluated constrained by the interference of the primary network. The authors show that the interference can be combated with the inclusion of more relays at the secondary network. In [26], the authors evaluate the outage probability and power allocation for a cognitive network with multiple primary transmitters and primary receivers; the secondary network is composed of a cooperative network with multiple relays, and the direct link at the secondary network is also considered. The number of primary users has a very severe impact on the performance of the proposed scheme. In [27], the outage probability is evaluated for a cognitive scenario where a relay helps the transmissions of the primary users as well as the secondary users. The results show that the outage probability decreases in comparison to schemes without cooperation in both networks. In [28], the authors evaluate two spectrally efficient schemes for a secondary cooperative network with multiple $(L)$ destinations, in which the best destination is selected. Such schemes consider incremental DF relaying protocol. The proposed schemes achieve full-diversity order, being equal to $L+1$.

Even though we acknowledge the extensive contributions of the previous works [18-28], it should be emphasized that they only considered HD relays, which is spectrally inefficient due to multiplexing loss. Thus, conversely to all previous works, in [29], the authors considered a scenario in which the secondary cooperative network utilizes a FD relay subject to self-interference. In [29], after matched filtering, the interference from the primary network is assumed to be seen as an additional Gaussian noise at the secondary one. Based on such assumption, [29] carried out a closed-form outage analysis for a FD dual-hop (FDDH) relaying scheme, in which the selfinterference at the relay was taken into account and the direct link was seen as interference by the secondary destination. 
In [30], in a non-cognitive radio scenario, the authors evaluate the outage probability of a cooperative network, where the signal of the direct link is exploited at the secondary destination and the relay employs the selectivedecode-and-forward (SDF) protocol. In [31], the authors evaluate the protocols proposed in [30], for a scenario subject to Nakagami-m fading. The proposed protocols have better performance in terms of outage probability than half-duplex and other full-duplex protocols. In [32], we evaluated the performance of the scheme proposed in [30] assuming a cooperative cognitive network operating in a spectrum sharing scenario with a FD relay subject to self-interference. However, the interference of the primary network into the secondary network was not considered in the analysis and the relay employed the fixed-decode-and-forward (FDF) protocol. Moreover, in [32], only equal power allocation (EPA) between source and relay is considered. The results show that the proposed joint-decoding full-duplex dual-hop secondary network outperforms its dual-hop full-duplex [29] and jointdecoding half-duplex counterparts, even in the presence of strong self-interference.

\subsection{Proposed scheme and summary of contributions}

In this paper, we consider a similar scenario as in [29, 32 ], in which the transmission through the direct link is exploited at the secondary destination and the interference of the primary network into the secondary network is taken into account. However, as in [30], the relay employs the SDF protocol. Additionally, an optimal power allocation (OPA) policy is proposed. The OPA strategy can choose between two modes of operation: (i) cooperation between source and relay or (ii) source transmission only. The proposed scheme, named as FD joint-decoding (FDJD) relaying, is compared with the FDDH scheme presented in [29] as well as with the standard HD relaying scheme. Closed-form expressions for the outage probability under EPA are derived and insightful discussions are drawn. Monte Carlo simulations are also presented in order to corroborate the proposed analysis.

Another contribution of this paper is to show that the proposed FDJD relaying scheme can considerably outperform the method proposed in [29] for the whole signalto-noise ratio (SNR) range. Our results also show that the proposed FDJD method performs better than the HD scheme even in the presence of strong self-interference.

The remainder of this paper is organized as follows: Section 2 introduces the system model, while in Section 3, an analytical performance analysis of the proposed scheme is carried out in terms of outage probability. In Section 4, an OPA strategy is introduced for the FDJD scheme. In Section 5, representative numerical results are provided and insightful discussions are drawn. Finally, Section 6 concludes the paper.

\subsubsection{Notations and functions}

Mathematical expectation is denoted by $\mathrm{E}[\cdot] ; \operatorname{Pr}[\cdot]$ stands for probability; $f_{W}(\cdot)$ and $F_{W}(\cdot)$ represent the probability density function (PDF) and cumulative distribution function (CDF) of a given random variable (RV) $W$, respectively.

\section{System model}

\subsection{Network model}

We consider a similar scenario as in [29, 32], with a cooperative cognitive network composed by one secondary transmitter $T_{s}$ (source), one FD secondary relay $r$, one secondary destination $D_{s}$ (destination), one primary transmitter $T_{p}$, and one primary destination $D_{p}$, as depicted in Fig. 1. The cognitive network operates in a spectrum sharing environment, where the secondary users are able to transmit their messages, if the interference on the primary network is below a certain threshold $I_{t h}[1-3]$. The transmit power constraints will be detailed later.

\subsection{Relay operation}

The main motivation behind FD schemes is the potential to overcome the spectral inefficiency of the HD ones, since the whole communication process occurs within one time slot. Nevertheless, such great potential comes at a price of self-interference, since it is a strenuous task to isolate transmit and received powers $[10,11]$. In the last few years, great efforts have been done on the mitigation of the self-interference [9-11]; however, these works also show that self-interference cannot be completely removed but considerably attenuated-even though employing sophisticated schemes of interference cancelation. Thus, there

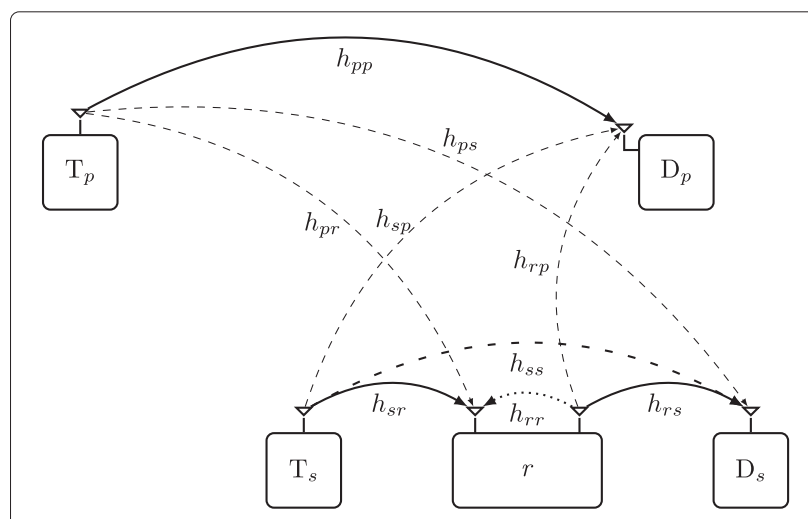

Fig. 1 System model: an underlay cooperative cognitive network with a FD relay suffering from self-interference and interference from the primary network 
remains residual self-interference at the relay, that is modeled as a fading channel which, by its turn, allows the emulation of various (non)line-of-sight configurations arising from antenna isolation and interference cancelation techniques $[9,10,15-17]$.

In FD mode, conversely to the HD schemes, the relay receives and transmits simultaneously in the same frequency band [8]. Thus, in a first phase-known as broadcast phase $(\mathrm{BC})$-the source broadcasts its message to the relay as well as to the destination. Simultaneously, the relay starts the decode-and-forward process-known as multiple access phase (MAC). In this second phase, the destination receives both signals from source and relay as in a multiple access channel [8].

\subsubsection{Encoding/decoding}

We rely on a variation of the DF scheme initially proposed in [33] and later extended to fading scenarios $[8,15]$. The DF protocol proposed in [33] is based on a combination of block Markov encoding at source and relay, associated with coding for cooperative multiple access channel and superposition coding $[8,33]$. As pointed out in [8], the same achievable rates for the DF protocol proposed in [33] - named irregular encoding/successive decoding [8] - can be achieved through different strategies: regular encoding/sliding-window decoding [34] and regular encoding/backward decoding [30, 35]. As indicated in [8], the latter is more suitable for quasi-static fading channels.

Therefore, hereafter, we assume regular encoding and backward decoding [30, 35]. Figure 2 depicts such encoding/decoding strategy where the frame is split into $L$ blocks. Notice that $x$ denotes the message sent by the source, while $\tilde{x}$ is the re-encoded message at the relay. There is a delay between source and relay transmissionwhich we assume to be of one block in this work. We recall that the backward decoding scheme is generalized in [30] for any number of delayed and total number of blocks. In that work, it is shown that performance is not affected as long as the ratio between delay and number of blocks is small.

\subsubsection{Selective-decode-and-forward}

In [30], the authors extended the traditional DF protocol to its variant, in which the relay only for wards the message if correctly decoded-such variant is named SDF, and is well known in the literature for the HD case [5]. Hereafter, we assume that the relay employs the SDF in FD mode. Further details are given later in Section 3.1.

\subsection{Channel model}

At time $t$, the received signals at the secondary relay and at the secondary destination are, respectively,

$$
\begin{aligned}
& y_{r}[t]=\sqrt{P_{s}} h_{s r} x[t]+\sqrt{P_{r}} h_{r r} \tilde{x}+\sqrt{P_{p}} h_{p r} x_{p}[t]+n_{r}[t], \\
& y_{d}[t]=\sqrt{P_{r}} h_{r s} \tilde{x}+\sqrt{P_{s}} h_{s s} x[t]+\sqrt{P_{p}} h_{p s} x_{p}[t]+n_{d}[t],
\end{aligned}
$$

where $P_{i}$ represents the transmit power, and indexes $i \in$ $\{p, s, r\}$ and $j \in\{r, s, p\}$ represent the transmitter/receivers, with $p$ denoting the primary transmitter or receiver, $s$ the secondary transmitter or receiver, and $r$ the relay. Note that $x[t]$ is the message sent by the source, and $\tilde{x} \triangleq x[t-D]$ is the message sent by $r$ after a processing delay $D$. Additionally, $x_{p}[t]$ is the message sent by $T_{p}$, and $n_{j}[t] \sim \mathcal{C N}\left(0, \sigma_{n}^{2}\right)$ stands for the complex additive white Gaussian noise at node $j$ with variance $\sigma_{n}^{2}=N_{0}$, where $N_{0}$ is the one-sided noise power spectral density. We assume hereafter to be $D=1$. The quasi-static fading channels are denoted by $h_{i j}$, and all channels undergo independent, identically distributed (i.i.d.) circularly symmetric complex Gaussian distribution with zero mean and variances $\sigma_{i j}$. The average fading power is $\lambda_{i j} \triangleq \mathbb{E}\left[\left|h_{i j}\right|^{2}\right] \triangleq d_{i j}^{-v} \sigma_{i j}$, where $d_{i j}$ represents the distance between nodes $i$ and $j$, and $v$ is the path-loss exponent $(v \geq 2)$. Moreover, as aforementioned, the residual self-interference is modeled as a fading channel such that $h_{r r} \sim \mathcal{C N}\left(0, \sigma_{r r}\right)$, with average fading power $\lambda_{r r} \triangleq P_{r} \delta \sigma_{r r}$, where $\delta$ represents the interference cancelation factor which arises from the association of antenna cancelation and interference cancelation techniques. Hereafter, for simplicity of notation, let us define $g_{i j} \doteq\left|h_{i j}\right|^{2}$.

As aforementioned, the message is divided into $L$ blocks, as shown in Fig. 2. Note that after the destination receives the $L$-th block from the relay, the destination is able to perform backward decoding and jointly decode the messages from source and relay $[8,30]$. As pointed out in [12], this delay is large enough to guarantee that the simultaneously received signals are uncorrelated, and therefore can be jointly decoded.

\begin{tabular}{c|c|c|c|c|c|c|}
\cline { 2 - 5 } Source & $x[1]$ & $x[2]$ & $\cdots$ & $x[L-1]$ & $x[L]$ & \\
\cline { 2 - 6 } Relay & & $\tilde{x}[1]$ & $\tilde{x}[2]$ & $\ldots$ & $\tilde{x}[L-1]$ & $\tilde{x}[L]$ \\
\hline
\end{tabular}

Fig. 2 FD relaying encoding. The message is split into $L$ blocks - note that the relay is one block delayed compared to the source. After receiving the last block from the relay, the destination applies backward decoding to jointly decode the messages 
Inspired by [29], after matched filtering, we can approximate the interference from the primary network by an additional noise, whose variance depend solely on the channel statistics of the primary network [29] — which can be estimated by the secondary network. This is a common assumption in the literature, as can be seen in [36-39]. Keeping this in mind, we can consider the primary interference as having a Gaussian distribution with zero mean and variance $\sigma_{p r}^{2}=P_{p} \lambda_{p r}$ at the relay and variance $\sigma_{p s}^{2}=P_{p} \lambda_{p s}$ at the destination. Then, according to ([29], Eq. 7), the overall Gaussian noise variance at the destination including the effect of the interference from the primary transmitter is $\sigma_{s}^{2}=\sigma_{n}^{2}+\sigma_{p s}^{2}$, while at relay, we have $\sigma_{r}^{2}=\sigma_{n}^{2}+\sigma_{p r}^{2}$.

\subsubsection{Spectrum sharing environment}

The primary receiver tolerates a maximum interference threshold given by $I_{t h}$. Since a FD relay is employed, the secondary transmitter and the secondary relay transmit their messages simultaneously, which means that primary destination receives interference from both secondary transmitters at the same time. Bearing this in mind, the transmission power of the secondary transmitter and the secondary relay must be constrained as [29]:

$$
g_{s p} P_{s}+g_{r p} P_{r} \leq I_{t h}
$$

In case of EPA, each transmitter has its transmission power constrained to half of the maximum interference threshold $I_{t h}$. Thus, the secondary transmitter and the secondary relay have their transmit powers limited, respectively, by [29]:

$$
P_{s}=\frac{I_{t h}}{2 g_{s p}}, \quad P_{r}=\frac{I_{t h}}{2 g_{r p}} .
$$

\section{Outage probability analysis}

In this section, we present the outage probability analysis, but first, let us define the attempted transmission rate and outage probability as follows.

Definition 1. Attempted transmission rate: the transmission rate in bits $/ \mathrm{s} / \mathrm{Hz}$, which is denoted by $\mathcal{R}$.

Definition 2. Outage probability: the probability of a failure in the communication between nodes $i$ and $j$ [40], so that an outage can be defined as the event that the mutual information between nodes $i$ and $j, \mathcal{I}_{i j}$, is lower than the attempted transmission rate $\mathcal{R}$ [40]. Assuming unitary bandwidth and Gaussian inputs, the outage probability is [40],

$$
\mathcal{O}_{i j}=\operatorname{Pr}\left[\mathcal{I}_{i j}<\mathcal{R}\right]=\operatorname{Pr}\left[\log _{2}\left(1+\frac{g_{i j} P_{i}}{N_{0}}\right)<\mathcal{R}\right] .
$$

Next, we introduce the proposed protocol-FDJD. We also include the outage formulations for the cases of the FDDH and HD schemes, which are already known $[14,15$, 29] and are used as benchmarks for the proposed scheme. The derivations are included for the sake of completeness.

\subsection{Full-duplex joint-decoding (FDJD)}

The proposed cooperative communication schemeFDJD - relies on the help of a FD relay such that the direct link is seen as useful information rather than interference at the destination. Thus, when the source-relay link is in outage, the message can still be successfully received through the direct link. Note that in the FDDH scheme [29], the direct link is never seen as useful information, but as interference at the destination. In the FDJD scheme, the direct link can be seen as useful information because we assume a more elaborated transmission scheme, as detailed in [30, 31] which makes use of backward decoding, that is superior than the one used in FDDH scheme [29].

First, the source broadcasts its message to relay and destination. Then, after a processing delay $D$, during the multiple access phase, the relay forwards the received message to the destination. Note that if the source-relay link is in outage, then the relay remains silent; otherwise, it forwards the message to the destination. By its turn, the destination jointly decodes the signals received from source and relay $[8,15,17,30]$, which is an iterative process dependent of block decoding $[8,30]$ - whose purposed is to combine the transmissions from source and relay-using advanced detection techniques and the acquired knowledge from previously received frames [30]. Additionally, based on [30] and in order to make a fair comparison with the schemes FDDH [29] and HD [5], we set $D=1$. In other words, there is a delay of one block among the messages sent by the secondary transmitter and the relay. Recall that small delays do not affect the overall performance of the FD protocol for a large $L$ $[8,30]$.

Next, we assess the overall mutual information of the FD scheme in the following lemma.

Lemma 1. The mutual information of the FDJD scheme is characterized as a function of the mutual information of the two decomposed phases: $B C$ and $M A C$, respectively, $\mathcal{I}_{B C}$ and $\mathcal{I}_{M A C}[8]$ and the mutual information of the direct link [30]. Thus,

$$
\mathcal{I}_{F D}=\max \left\{\mathcal{I}_{S S}, \min \left\{\mathcal{I}_{B C}, \mathcal{I}_{M A C}\right\}\right\}
$$

Proof. Under the SDF protocol, the transmission rate is maximized accordingly to the direct link or to the cooperative link as introduced in [5] for the HD schemes. Under 
FD mode, the authors in [30] show that the mutual information can be written in similar fashion when backward decoding is applied.

Thus, from Lemma 1 and in the context of a cognitive cooperative network and relying on $[8,15,17,30]$, we can write the mutual information of the $\mathrm{BC}$ phase of the FDJD scheme as

$$
\begin{aligned}
& \mathcal{I}_{s r}^{\text {ID }}=\log _{2}\left(1+\frac{P_{s} g_{s r}}{P_{r} g_{r r}+\sigma_{r}^{2}}\right), \\
& \mathcal{I}_{s s}^{\text {ID }}=\log _{2}\left(1+\frac{P_{s} g_{s s}}{\sigma_{s}^{2}}\right),
\end{aligned}
$$

while the mutual information between relay and destination, in the MAC phase, is

$$
\mathcal{I}_{r s}^{\mathrm{ID}}=\log _{2}\left(1+\frac{P_{s} g_{s s}+P_{r} g_{r s}}{\sigma_{s}^{2}}\right) .
$$

Note that in (9), the signals coming from source and relay are seen as useful information by the destination. Moreover, also note that the self-interference at the relay is taken into account in (7). Next, given Definition 2, the overall outage probability of the FDJD scheme is given as follows.

Theorem 1. Given Definition 2 and Lemma 1, the overall outage probability of the proposed FDJD scheme is

$$
\begin{aligned}
\mathcal{O}_{J D} & =\operatorname{Pr}\left[\mathcal{I}_{F D}<\mathcal{R}\right] \\
& =\mathcal{O}_{s r}^{I D} \mathcal{O}_{s s}^{J D}+\left(1-\mathcal{O}_{s r}^{J D}\right) \mathcal{O}_{r s}^{J D} .
\end{aligned}
$$

Proof. All RVs are i.i.d., which allows us to write the overall outage probability as a function of the outage probabilities of the individual links. Moreover, from [15, 30], we know that the outage event from the backward decoding of source and relay messages can be represented by $\mathcal{O}_{r s}^{\text {D }}$, which includes the joint-decoding processes at the destination.

In order to assess (10) in Theorem 1, we investigate next the outage probability of the individual links.

Lemma 2. Assuming that all RVs are i.i.d and exponentially distributed with rate $\lambda_{i j}^{-1}$, the outage probability of the link between the relay and the secondary destination $\mathcal{O}_{r s}^{I D}$ is given by

$$
\begin{aligned}
\mathcal{O}_{r s}^{I D}= & \lambda_{r p} \lambda_{s p} \frac{\epsilon\left(\epsilon \lambda_{r p} \lambda_{s p}+\lambda_{r p} \lambda_{s s} \mu_{s}+\lambda_{r s} \lambda_{s p} \mu_{s}\right)}{\left(\epsilon \lambda_{r p} \lambda_{s p}+\lambda_{r p} \lambda_{s s} \mu_{s}+\lambda_{r s} \lambda_{s p} \mu_{s}\right)^{2}} \\
& -\frac{\lambda_{r p} \lambda_{s p} \lambda_{r s} \lambda_{s s} \mu_{s}^{2} \ln \left(\frac{\left(\epsilon \lambda_{r p}+\lambda_{r s} \mu_{s}\right)\left(\epsilon \lambda_{s p}+\lambda_{s s} \mu_{s}\right)}{\lambda_{r s} \lambda_{s s} \mu_{s}^{2}}\right)}{\left(\epsilon \lambda_{r p} \lambda_{s p}+\lambda_{r p} \lambda_{s s} \mu_{s}+\lambda_{r s} \lambda_{s p} \mu_{s}\right)^{2}},
\end{aligned}
$$

where $\epsilon=2^{\mathcal{R}}-1$ and $\mu_{s}=I_{t h} /\left(2 \sigma_{s}^{2}\right)$.
Proof. Let us define $Z_{k l}$ as $Z_{k l}=g_{k l} / g_{k p}$, with $k=$ $\{s, r\}$ and $l=\{s, r\}$ whose PDF and CDF are given in Appendix 1. Let us also define $W=Z_{s s}+Z_{r s}$ whose $\operatorname{PDF} f_{W}(w)$ and $\operatorname{CDF} F_{W}(w)$ are introduced in Appendix 2. Bearing this in mind, the outage probability of the link between relay and destination can be written as

$$
\begin{aligned}
\mathcal{O}_{r s}^{\text {ID }} & =\operatorname{Pr}\left[\mathcal{I}_{r s}^{\mathrm{ID}}<\mathcal{R}\right] \\
& =\operatorname{Pr}\left[Z_{s s}+Z_{r s}<\frac{\epsilon}{\mu_{s}}\right] \\
& =\operatorname{Pr}\left[W<\frac{\epsilon}{\mu_{s}}\right] \\
& =F_{W}\left(\frac{\epsilon}{\mu_{s}}\right) .
\end{aligned}
$$

Thus, after a few algebraic manipulations into (15), we attain (11), which concludes the proof.

Following [29], the outage probability of the sourcerelay link is given by

$$
\begin{aligned}
\mathcal{O}_{s r}^{\mathrm{ID}}= & \epsilon \lambda_{s p} \frac{\left(\lambda_{r p}-\lambda_{r r} \mu_{r}\right)\left(\lambda_{r p} \lambda_{s p} \epsilon+\lambda_{r p} \lambda_{s r} \mu_{r}-\lambda_{r r} \lambda_{s p} \epsilon \mu_{r}\right)}{\left(\lambda_{r p} \lambda_{s p} \epsilon+\lambda_{r p} \lambda_{s r} \mu_{r}-\lambda_{r r} \lambda_{s p} \epsilon \mu_{r}\right)^{2}} \\
+ & \frac{\epsilon \lambda_{s p} \lambda_{r p} \lambda_{r r} \lambda_{s r} \mu_{r}^{2} \ln \left(\frac{\lambda_{r p}\left(\lambda_{s p} \epsilon+\lambda_{s r} \mu_{r}\right)}{\lambda_{r r} \lambda_{s p} \epsilon \mu_{r}}\right)}{\left(\lambda_{r p} \lambda_{s p} \epsilon+\lambda_{r p} \lambda_{s r} \mu_{r}-\lambda_{r r} \lambda_{s p} \epsilon \mu_{r}\right)^{2}}
\end{aligned}
$$

where $\epsilon=2^{\mathcal{R}}-1$ and $\mu_{r}=I_{t h} / 2 \sigma_{r}^{2}$.

Remark 1. With the objective of obtaining a more general approach, we consider, differently of [29], that the channel between the source and the relay has average fading power $\lambda_{s r}$ different of one.

The outage probability of the source-destination link is [41]

$$
\begin{aligned}
\mathcal{O}_{s s}^{\text {ID }} & =\operatorname{Pr}\left[\mathcal{I}_{s s}^{J D}<\mathcal{R}\right] \\
& =\frac{\lambda_{s p} \epsilon}{\lambda_{s p} \epsilon+\lambda_{s s} \mu_{s}},
\end{aligned}
$$

where $\epsilon=2^{\mathcal{R}}-1$ and $\mu_{s}=I_{t h} /\left(2 \sigma_{s}^{2}\right)$.

Finally, the overall outage probability of the FDJD scheme can be attained by plugging (11), (16), and (18) into (10).

\subsection{Full-duplex dual hop (FDDH)}

Differently from the proposed FDJD scheme, in the FDDH scheme, the direct link is seen as interference at the destination [29].

Notice that the mutual information of the source-relay link is written as in (7). However, differently from (9), here the source transmission is seen as interference. Thus, 
the the mutual information of the relay-destination link becomes

$$
\mathcal{I}_{r s}^{\mathrm{DH}}=\log _{2}\left(1+\frac{g_{r s} P_{r}}{g_{s s} P_{s}+\sigma_{s}^{2}}\right) .
$$

The overall outage probability of the FDDH scheme is $[15,29]$

$$
\begin{aligned}
\mathcal{O}_{\mathrm{DH}} & =\operatorname{Pr}\left[\min \left\{\mathcal{I}_{s r}^{\mathrm{DH}}, \mathcal{I}_{r s}^{\mathrm{DH}}\right\}<\mathcal{R}\right] \\
& =\mathcal{O}_{s r}^{\mathrm{DH}}+\mathcal{O}_{r s}^{\mathrm{DH}}-\mathcal{O}_{s r}^{\mathrm{DH}} \mathcal{O}_{r s}^{D H} .
\end{aligned}
$$

Additionally, $\mathcal{O}_{s r}^{\mathrm{DH}}=\operatorname{Pr}\left[\mathcal{I}_{s r}^{\mathrm{DH}}<\mathcal{R}\right]$ is written as in (16). Notice that $\mathcal{O}_{r s}^{\mathrm{DH}}=\operatorname{Pr}\left[\mathcal{I}_{r s}^{\mathrm{DH}}<\mathcal{R}\right]$ is also given as in (16) but with the following substitutions: $\lambda_{r r}$ by $\lambda_{s s}, \lambda_{s r}$ by $\lambda_{r s}$, $\lambda_{s p}$ by $\lambda_{r p}$, and $\lambda_{r p}$ by $\lambda_{s p}$.

\subsection{Half-duplex (HD)}

Differently from FD schemes, in the HD mode, the secondary transmissions occur within two time slots according to the SDF protocol [5], therefore incurring in multiplexing loss. In the first time slot, the source broadcasts its message, while in the second time slot, the relay acts only if the source message was decoded free of error. Thus, at the destination, both messages are combined and jointly decoded-which can be seen as maximal ratio combining [5].

Finally, since only one transmission per time slot occurs in the HD scheme, the transmission power of the secondary transmitter and of the relay are constrained, respectively as:

$$
\begin{aligned}
& P_{s}=\frac{I_{t h}}{g_{s p}}, \\
& P_{r}=\frac{I_{t h}}{g_{r p}} .
\end{aligned}
$$

The overall outage probability of the HD scheme can be finally defined as $[7,29]$

$$
\mathcal{O}_{H D}=\mathcal{O}_{s s}^{H D} \mathcal{O}_{s r}^{H D}+\left(1-\mathcal{O}_{s r}^{H D}\right) \mathcal{O}_{r s}^{H D} .
$$

The outage probability $\mathcal{O}_{r s}^{\mathrm{HD}}$ is written as in (11), but replacing $\epsilon$ by $\varepsilon$. The outage probability of the source-relay link can be expressed as [41]

$$
\mathcal{O}_{s r}^{\mathrm{HD}}=\frac{\lambda_{s p} \varepsilon}{\lambda_{s p} \varepsilon+2 \lambda_{s r} \mu_{r}},
$$

while the outage probability of the source-destination link is given by (24), but replacing $\mu_{r}$ by $\mu_{s}$ and $\lambda_{s r}$ by $\lambda_{s s}$, with $\varepsilon=2^{2 \mathcal{R}}-1$.

\section{Power allocation policies}

In this section, we propose an OPA for the proposed FDJD cognitive network. We consider that in an OPA policy, the secondary nodes may obtain the information of the channels to the primary network by direct feedback from the primary receiver [3].
Moreover, we recall that under the FDJD scheme, the direct link is seen as a useful source of information, and therefore the proposed power allocation strategy can choose between two modes of operation: (i) cooperation between source and relay or (ii) source transmission only.

In order to achieve the maximal performance, we attempt to minimize the overall outage probability or equivalently to maximize the signal-to-interference-plusnoise ratio (SINR) of the secondary network. Thus, as we consider that the system operates in one of two modes (cooperation or source transmission only), the optimization problem can be formulated as

$$
\begin{array}{r}
\max \left\{\gamma_{s s}, \gamma_{\text {coop }}\right\}, \\
\text { s.t. } \quad g_{s p} P_{s}^{\star}+g_{r r} P_{r}^{\star}<I_{t h}, \\
P_{s}^{\star}, P_{r}^{\star} \geq 0 .
\end{array}
$$

where $\gamma_{\text {coop }}=\min \left\{\gamma_{s r}, \gamma_{J D}\right\}$ is the SINR when the cooperation is favorable. In this case, $\gamma_{I D}$ is the SINR of the virtual link between source, relay, and destination as seen at the destination after joint-decoding.

\subsection{OPA policy for FDJD relaying}

The proposed cooperative cognitive network may operate in one of two modes:

1. The relay cooperates with the secondary transmission and the destination jointly decodes the signals from source and relay. For the cooperative case, the maximum of $\gamma_{\text {coop }}$ occurs when the links between source and relay, and the virtual link between source, relay, and destination present the same achievable rate. If one of the links works with greater achievable rate than the other, there is an excess of the SINR in one of the links and consequently a waste of power that could be allocated to increase the achievable rate of the weaker link. In order to ensure that two links achieve the same rate, we must enforce that the SINRs seen at the relay and at the destination are equal. We consider that the channels remain constant for at least $L+D$ time slots, to ensure the optimal power allocation.

2. If the equivalent SINR with the two links working with the same capacity is lower than the SINR when the source communicates with the destination without the help of the relay, the secondary source transmits the message without the help of the relay. In this case, the whole available power is allocated to the source and the relay is momentarily put in idle mode. 
Next, we define the SINR of the links $\gamma_{s r}, \gamma_{s s}$, and $\gamma_{J D}$, as

$$
\begin{aligned}
\gamma_{s s} & =\frac{g_{s s} P_{s}^{\star s s}}{\sigma_{s}^{2}} \\
\gamma_{s r} & =\frac{g_{s r} P_{s}^{\star}}{g_{r r} P_{r}^{\star}+\sigma_{r}^{2}} \\
\gamma_{\mathrm{ID}} & =\frac{g_{r s} P_{r}^{\star}+g_{s s} P_{s}^{\star}}{\sigma_{s}^{2}},
\end{aligned}
$$

where $P_{r}^{\star}$ and $P_{s}^{\star}$, respectively, are the optimal transmit powers for the relay and the secondary transmitter when the cooperation is favorable. The transmit power for the secondary transmitter when the relay is turned off is represented by $P_{s}^{\star s}$. We recall that after the whitening filter, the noise powers at source and relay are, respectively, $\sigma_{s}^{2}=\sigma_{n}^{2}+\sigma_{p s}^{2}$ and $\sigma_{r}^{2}=\sigma_{n}^{2}+\sigma_{p r}^{2}$.

When cooperation is favorable, it follows that $\gamma_{\text {coop }}>$ $\gamma_{s s}$, which allows us to rearrange the optimization problem as

$$
\begin{aligned}
\frac{g_{s r} P_{s}^{\star}}{g_{r r} P_{r}^{\star}+\sigma_{r}^{2}} & =\frac{g_{r s} P_{r}^{\star}+g_{s s} P_{s}^{\star}}{\sigma_{s}^{2}} \\
g_{s p} P_{s}^{\star}+g_{r p} P_{r}^{\star} & =I_{t h .} .
\end{aligned}
$$

In the following, we introduce a procedure that calculates the OPA for the FDJD scheme as shown by Algorithm 1 . Notice that the relay is put in idle mode if direct transmission is more favorable. Moreover, by Algorithm 1, we observe that first, the transmit powers assuming cooperative and non-cooperative transmissions are calculated, and then the SINRs $\gamma_{\text {coop }}$ and $\gamma_{s s}$ are compared.

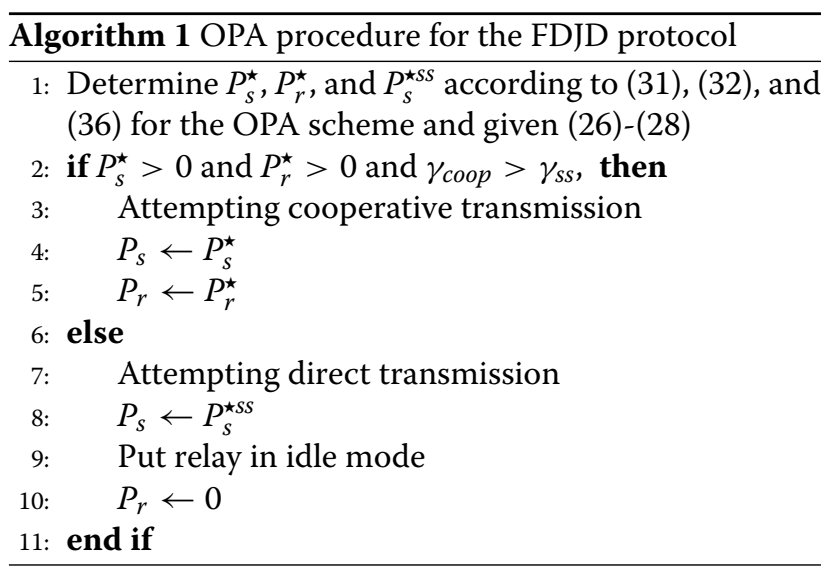

Theorem 2. Assuming that source and relay cooperate under the FDJD protocol, the solution of the system of equations-composed of (29) and (30)-gives the source's optimal transmit power as

$$
P_{s}^{\star}= \begin{cases}-\frac{1}{2 \alpha}\left(\beta+\sqrt{\beta^{2}-4 \alpha \kappa}\right) & 0<g_{s s}<\frac{g_{s p} g_{r s}}{g_{r p}} \\ \frac{1}{2 \alpha}\left(-\beta+\sqrt{\beta^{2}-4 \alpha \kappa}\right) & g_{s s}>\frac{g_{s p} g_{r s}}{g_{r p}}\end{cases}
$$

while the relay's optimal transmit power is

$$
P_{r}^{\star}=\frac{I_{t h}-P_{s}^{\star} g_{s p}}{g_{r p}},
$$

where

$$
\begin{aligned}
\alpha= & g_{r s} g_{r r} g_{s p}^{2}-g_{s s} g_{r r} g_{s p} g_{r p} \\
\beta= & g_{r r} g_{s s} g_{r p} I_{t h}-2 g_{r r} g_{s p} g_{r s} I_{t h} \\
& -g_{r p}^{2}\left(g_{s r} \sigma_{s}^{2}-g_{s s} \sigma_{r}^{2}\right)-g_{r p} g_{s p} g_{r s} \sigma_{r}^{2} \\
\kappa= & I_{t h}\left(g_{r r} g_{r s} I_{t h}+g_{r p} g_{r s} \sigma_{r}^{2}\right) .
\end{aligned}
$$

Proof. Isolating $P_{r}^{\star}$ from (30) and putting it into (29), we attain a quadratic equation, whose roots give (31). Once $P_{s}^{\star}$ is attained, we readily obtain (32) from (30).

On the other hand, when the cooperation is not favorable such that $\gamma_{\text {coop }}<\gamma_{s s}$ or $P_{s}^{\star}<0$ or $P_{r}^{\star}<0$, the relay is turned off, remaining idle until the next time slot when the process is repeated.

When cooperation is not viable, the secondary transmitter optimally allocates its power according to

$$
P_{s}^{\star s s}=\frac{I_{t h}}{g_{s p}} .
$$

Further, once the optimal transmit powers is attained, we can assess the outage probability under OPA policy as follows.

Theorem 3. Assuming OPA policy, the overall outage probability of the FDJD schemes is

$$
\mathcal{O}_{\text {OPA }}= \begin{cases}\mathcal{O}_{\text {ss }} \mathcal{O}_{\text {coop }} & \gamma_{\text {coop }}>\gamma_{s s} \\ \mathcal{O}_{s s} & \text { otherwise }\end{cases}
$$

Proof. Cooperation only happens if $\gamma_{\text {coop }}>\gamma_{s s}$, and since symmetric rates in the SR and in the virtual JD link maximize $\gamma_{\text {coop }}$, the outage probability becomes

$$
\begin{aligned}
\mathcal{O}_{\text {coop }} & \left.=\operatorname{Pr}\left[\log _{2}\left(1+\gamma_{\text {coop }}\right)<\mathcal{R}\right)\right] \\
& \left.=\operatorname{Pr}\left[\log _{2}\left(1+\gamma_{\mathrm{JD}}\right)<\mathcal{R}\right)\right],
\end{aligned}
$$

where $\gamma_{\mathrm{ID}}$ is given in (28). On the other hand, when the relay is put in idle mode, the outage probability becomes

$$
\mathcal{O}_{s s}=\operatorname{Pr}\left[\log _{2}\left(1+\gamma_{s s}\right)<\mathcal{R}\right],
$$

where $\gamma_{s s}$ is given in (26). 


\subsection{OPA policy for FDDH relaying}

Now, we investigate the optimization problem for the FDDH scheme, proposed in [29]. The optimization problem can be written as

$$
\begin{gathered}
\min _{P_{s}, P_{r} \geq 0} \mathcal{O}_{D H}=\operatorname{Pr}\left[\min \left\{\gamma_{s r}, \gamma_{\mathrm{DH}}\right\}<\epsilon\right], \\
\text { s.t. } \quad g_{s p} P_{s}+g_{r p} P_{r}<I_{t h} .
\end{gathered}
$$

The outage probability for the FDDH scheme is dominated by the link with the worst SINR. Therefore, the outage probability is minimized when the SINR at the relay $\gamma_{s r}$-which is given as in (27) - is equal to the SINR at the destination $\gamma_{\mathrm{DH}}$, which is given as

$$
\gamma_{\mathrm{DH}}=\frac{g_{r s} P_{r}^{\star}}{g_{s s} P_{s}^{\star}+\sigma_{s}^{2}} .
$$

Following [29], the optimal transmit power at source is given by

$$
P_{s}^{\star}=\frac{1}{2 \alpha}\left(-\beta+\sqrt{\beta^{2}-4 \alpha \kappa}\right)
$$

while the optimal transmit power of the relay is

$$
P_{r}^{\star}=\frac{I_{t h}-P_{s}^{\star} g_{s p}}{g_{r p}},
$$

where

$$
\begin{aligned}
\alpha & =g_{s s} g_{s r} g_{r p}^{2}-g_{r r} g_{s p}^{2} g_{r s} \\
\beta & =2 g_{r r} g_{s p} g_{r s} I_{t h}+g_{r p}^{2} g_{s r} \sigma_{s}^{2}+g_{r p} g_{s p} g_{r s} \sigma_{r}^{2} \\
\kappa & =-I_{t h}\left(g_{r r} g_{r s} I_{t h}+g_{r p} g_{r s} \sigma_{r}^{2}\right) .
\end{aligned}
$$

The outage probability of the FDDH scheme, $\mathcal{O}_{D H}$, under OPA policy is [29]

$$
\mathcal{O}_{D H}=\operatorname{Pr}\left[\log _{2}\left(1+\gamma_{D H}\right)<\mathcal{R}\right] .
$$

\section{Numerical results and discussions}

In this section, some numerical representative results along with insightful discussions regarding the performance of the proposed scheme are presented. Unless stated otherwise, for the plots we assume-without loss of generality-path-loss model $d_{i j}^{-v}$ with exponent $v=4$, where $d_{s r}=\frac{1}{4}, d_{s p}=1, d_{s s}=\frac{1}{2}, d_{r s}=\frac{1}{4}, d_{r p}=1, \sigma_{r r}=1$, $\delta=-40 \mathrm{~dB}, \mathcal{R}=4 \mathrm{bpcu}, P_{p}=10 \mathrm{~dB}$, and $N_{0}=1$. We also consider that the relay is positioned in a straight line between source and destination.

Figure 3 depicts the outage probability of the EPA for the FDJD scheme as a function of the interference threshold $I_{t h}$ imposed by the primary network. We also account for different levels of self-interference $\delta \in$ $\{0,-10,-20,-60\} \mathrm{dB}$, and assume $P_{p}=5 \mathrm{~dB}$ and $\mathcal{R}_{s}=$ $6 \mathrm{bpcu}$. Monte Carlo simulations regarding the EPA policy are represented by red circles. Notice that the simulations agree very well with the analytic results. Moreover, as we can see from Fig. 3, FD relaying is feasible once the performance increases with the increase of the quality of the

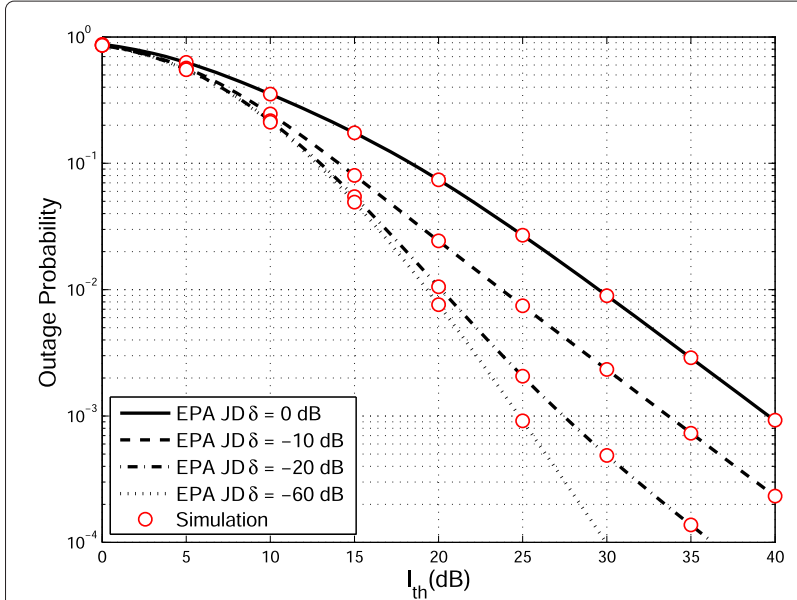

Fig. 3 Outage probability of the EPA FDJD scheme as a function of the primary interference threshold $I_{\text {th }}$, for different values of $\delta$, with $P_{p}=5 \mathrm{~dB}$ and $\boldsymbol{\mathcal { R }}=6 \mathrm{bpcu}$

interference cancelation at the relay, which is reflected in low values for $\delta$.

Figure 4 presents the outage probability as a function of the primary interference threshold $I_{t h}$ such that the proposed schemes are compared to FDDH [29], considering EPA and OPA, and to the half-duplex HD method. The proposed EPA FDJD scheme outperforms even the OPA FDDH proposed by [29]. Such performance gain comes from the additional diversity seen by the destination because the direct link is seen as useful information rather than interference. Furthermore, the proposed OPA FDJD has the best performance among all schemes, and we observe a larger diversity gain in comparison with FDDH

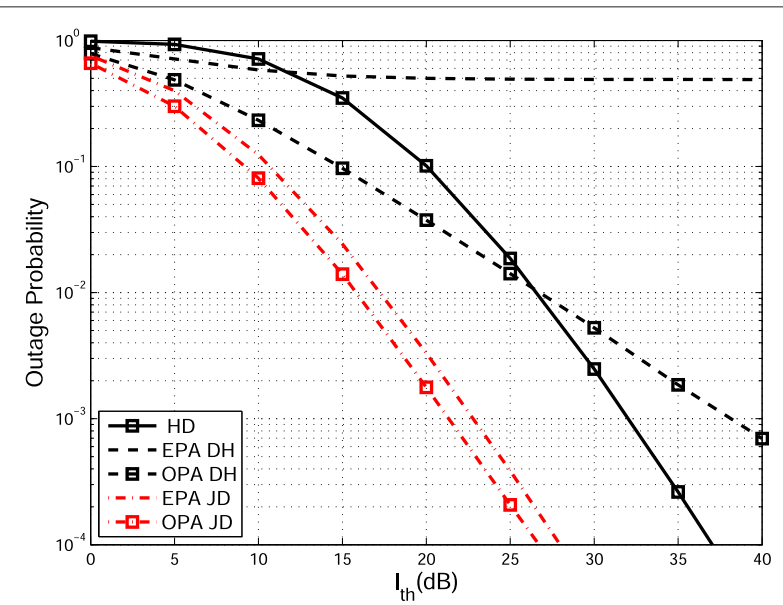

Fig. 4 Outage probability for the different schemes as a function of the primary interference threshold $I_{\text {th }}$, with $\mathcal{R}=4 \mathrm{bpcu}, P_{p}=10 \mathrm{~dB}$, and $\delta=-40 \mathrm{~dB}$ 
Next, Fig. 5 evaluates the outage probability for the EPA and OPA schemes as a function of the attempted transmission rate $\mathcal{R}$. Note that for low values of $\mathcal{R}$, the HD scheme has better performance than the FDDH schemes because of the performance floor caused by the selfinterference at the FD relay for high values of $I_{t h}$. Moreover, the proposed OPA FDJD has the best performance among all schemes for the whole $\mathcal{R}$ range, which means that under OPA policy, the FDJD protocol can operate at a much higher rate given an outage probability threshold.

Figure 6 compares the outage probability for the different schemes with the relay near the secondary transmitter. In this case, we consider $d_{s r}=\frac{1}{8}, d_{s s}=\frac{1}{2}, d_{r s}=\frac{3}{8}$, $d_{s p}=1, d_{r p}=1, d_{p r}=1, d_{p s}=1, \mathcal{R}=4 \mathrm{bpcu}, P_{p}=10$ $\mathrm{dB}$, and $\delta=-40 \mathrm{~dB}$. On the other hand, Fig. 7 considers the relay near the secondary destination. In this case, $d_{s r}=\frac{3}{8}, d_{s s}=\frac{1}{2}, d_{r s}=\frac{1}{8}$. From the figures, we can see that regardless of the relay positioning, the proposed FDJD scheme has the best performance among all the schemes considered.

Figure 8 compares the outage probability versus the primary transmit power $P_{p}$. We consider $d_{s r}=\frac{1}{4}, d_{s s}=$ $\frac{1}{2}, d_{r s}=\frac{1}{4}, d_{s p}=1, d_{r p}=1, d_{p r}=1, d_{p s}=1, \mathcal{R}=4 \mathrm{bpcu}$, $I_{\text {th }}=10 \mathrm{~dB}$, and $\delta=-40 \mathrm{~dB}$. From the figure, we can see that the OPA FDJD scheme outperforms the others schemes, even though suffering strong interference from the primary network.

In the following, Fig. 9 compares the percentage of power that is allocated for the secondary transmitter and for the relay as a function of the primary interference threshold $I_{t h}$ for three different positions of the relay: (a) exactly in between the secondary transmitter and destination $\left(d_{s r}=\frac{1}{4}, d_{s s}=\frac{1}{2}, d_{r s}=\frac{1}{4}\right)$; (b) near to secondary transmitter $\left(d_{s r}=\frac{1}{8}, d_{s s}=\frac{1}{2}, d_{r s}=\frac{3}{8}\right)$; and (c) near to secondary destination $\left(d_{s r}=\frac{3}{8}, d_{s s}=\frac{1}{2}, d_{r s}=\frac{1}{8}\right)$. In (a),

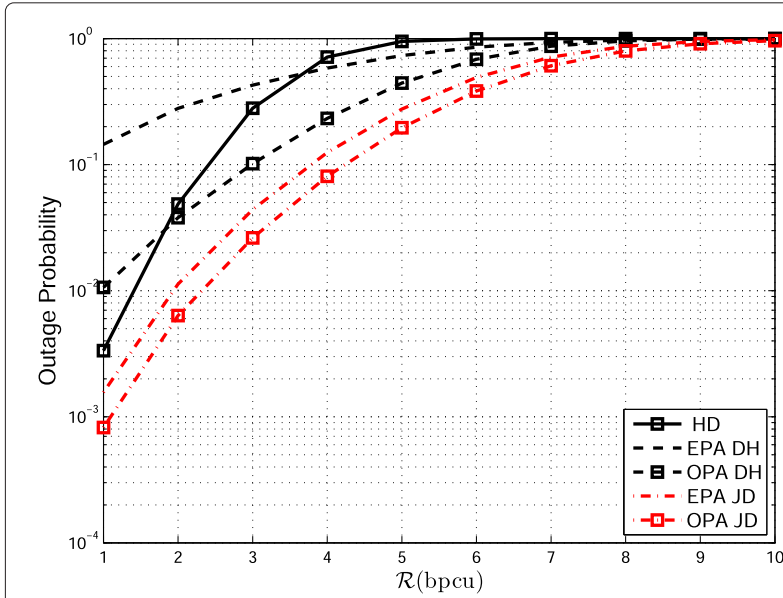

Fig. 5 Outage probability for the different schemes as a function of the attempted rate $\mathcal{R}$, with $P_{p}=10 \mathrm{~dB}, \delta=-40 \mathrm{~dB}$, and $l_{\text {th }}=10 \mathrm{~dB}$

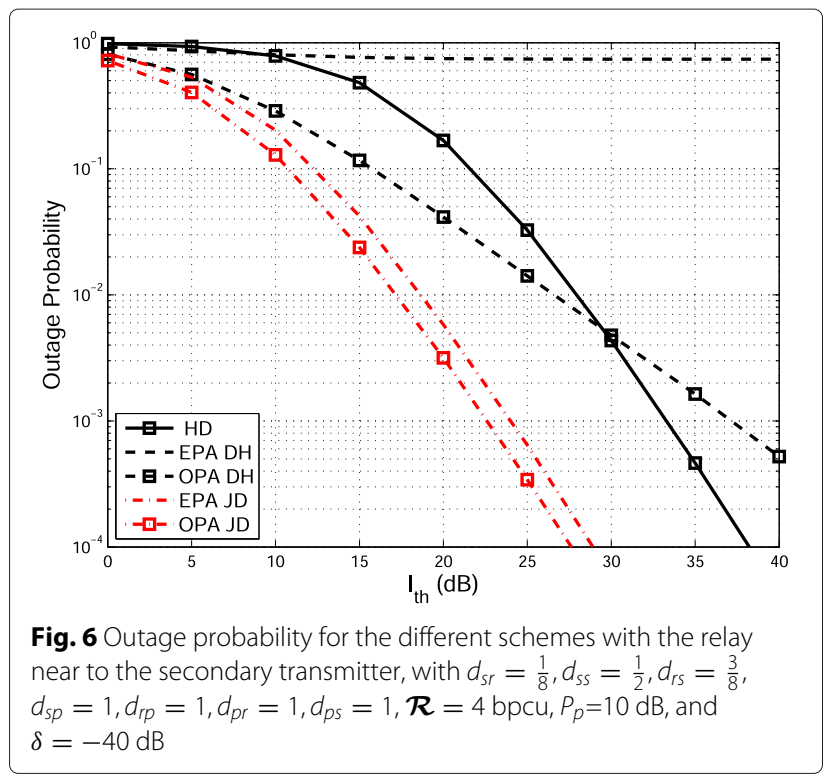

the FDJD scheme allocates more power for the secondary transmitter than for the relay. Recall that in FDJD, the direct link is seen as a useful source of information and also the relay can be put in idle mode, differently from the FDDH scheme where the relay is always fully operational. This also explains the fact that under the FDDH protocol, more power is allocated to the relay than to the source. While in (b), the OPA FDJD scheme allocates more power for the relay than in (a); once in this case, cooperation is preferred over direct transmission because the relay is closer to the source. We recall that the source-relay link is the bottleneck of cooperative relaying schemes; since the source and relay are closer, less outage events

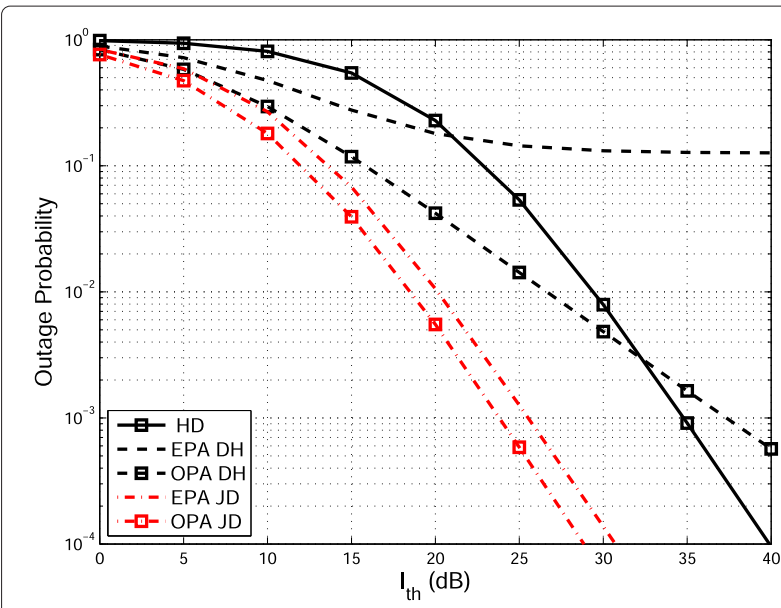

Fig. 7 Outage probability for the different schemes with the relay near to the secondary destination, with $d_{s r}=\frac{3}{8}, d_{s s}=\frac{1}{2}, d_{r s}=\frac{1}{8}$, $d_{s p}=1, d_{r p}=1, d_{p r}=1, d_{p s}=1, \mathcal{R}=4$ bpcu, $P_{p}=10 \mathrm{~dB}$, and $\delta=-40 \mathrm{~dB}$ 


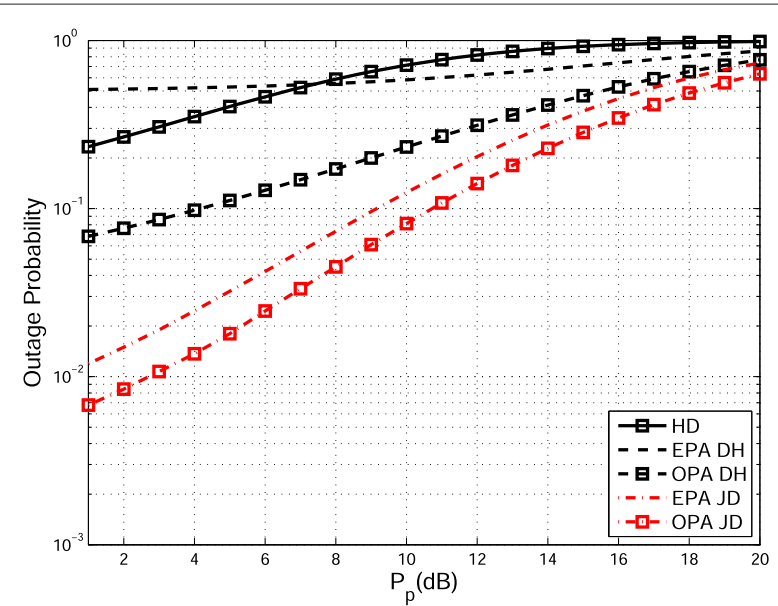

Fig. 8 Outage probability as a function of the primary transmit power $P_{p}$, with $d_{s r}=\frac{1}{4}, d_{s s}=\frac{1}{2}, d_{r s}=\frac{1}{4}, d_{s p}=1, d_{r p}=1, d_{p r}=1, d_{p s}=1$, $\mathcal{R}=4 \mathrm{bpcu}, I_{\text {th }}=10 \mathrm{~dB}$, and $\delta=-40 \mathrm{~dB}$

may occur which turns cooperation favorable. Next, in (c) and conversely to (b), the OPA FDJD strategy allocates more power for the secondary transmitter than the relay because the SINR $\gamma_{\text {coop }}$ is similar to the SINR of the direct link.

\subsection{Throughput analysis}

In this subsection, the spectrum efficiency of the fullduplex schemes are compared. The throughput of the full-duplex scheme sch $\in$ (EPA FDDH, OPA FDDH, EPA FDJD, OPA FDJD $\}\left(T_{\text {sch }}\right)$ is given by $T_{\text {sch }}=\mathcal{R}\left(1-\mathcal{O}_{\text {sch }}\right)$, where the outage probabilities of EPA and OPA FDJD schemes are given by (10) and (37), while the outage probabilities for EPA and OPA FDDH schemes are written as in (20) and (48).

In Fig. 10, we present the throughput $T_{\text {sch }}$ as a function of the primary interference threshold $I_{t h}$ for the FDJD and FDDH schemes, with $\mathcal{R}=4 \mathrm{bpcu}, P_{p}=10 \mathrm{~dB}$, and $\delta=$
$-40 \mathrm{~dB}$. From this figure, we can see that the proposed FDJD scheme has the best performance. Furthermore, the throughput of EPA DH saturates for $I_{t h}>20 \mathrm{~dB}$. This is caused by the increment of the self-interference at the relay and the interference of the direct link. Notice that even though suffering similar self-interference levels as the EPA DH, both JD protocols are able to decrease the effects of the self-interference.

\section{Conclusions}

This paper proposes a new FD scheme for a cognitive underlay network subject to self-interference as well as primary interference. The secondary destination jointly decodes the signals from the secondary transmitter and the relay-which differs from other works proposed in the literature where the direct link is seen as interference rather than useful information. We introduced new closed-form expressions for the outage probability of the proposed FD cooperative cognitive network under EPA policy. We first evaluated the FD schemes under EPA policy and show the feasibility of FD relaying under cognitive constraints. Our results showed that the proposed FDJD scheme considerably outperforms the known FDDH scheme and can operate at a much higher rate regime than other schemes. We propose a new optimal power allocation policy for the FDJD scheme. The OPA strategy can choose between two modes of operation, cooperation between source and relay or source transmission only. Our results show that the FDJD scheme under the proposed OPA policy presents the best performance among all schemes investigated in this paper.

\section{Appendices}

\section{Appendix 1: PDF and CDF of $Z_{k l}$}

Let $Z_{k l}$ be a RV defined as $Z_{k l}=g_{k l} / g_{k p}$, where $g_{i j} \sim$ $\operatorname{Exp}\left(\lambda_{i j}\right)$ and $k=\{s, r\}$ and $l=\{s, r\}$. Then, based on standard statistical methods [42], we readily attain the PDF of
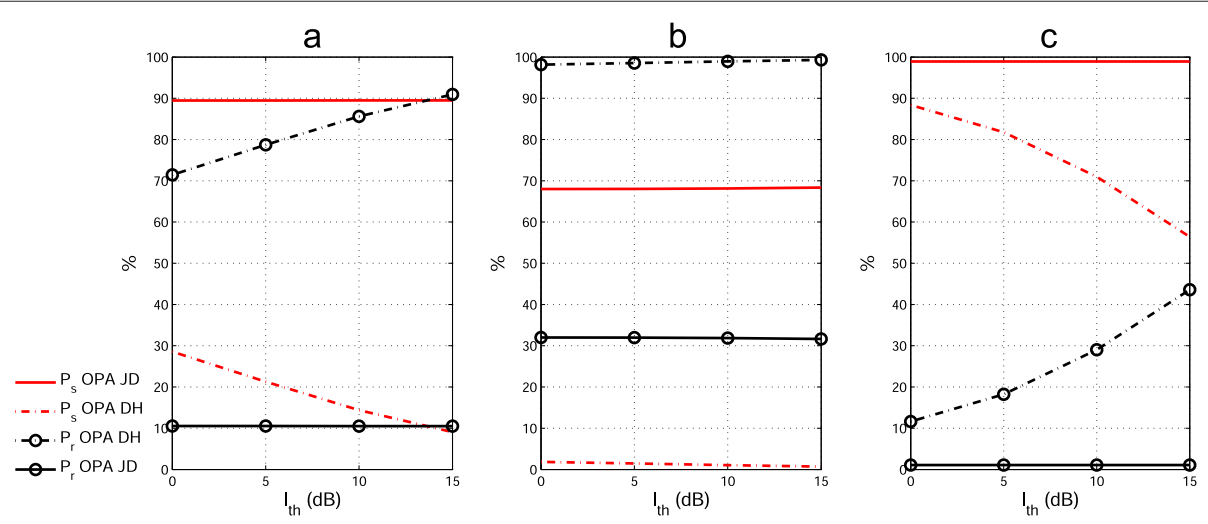

Fig. 9 Power allocation for $P_{s}$ and $P_{r}$ for three different positions of the relay: a exactly in between the secondary transmitter and destination, $\mathbf{b}$ near to secondary transmitter, and $\mathbf{c}$ near to secondary destination. Assume $\mathcal{R}=4 \mathrm{bpcu}, P_{p}=10 \mathrm{~dB}$, and $\delta=-40 \mathrm{~dB}$ 


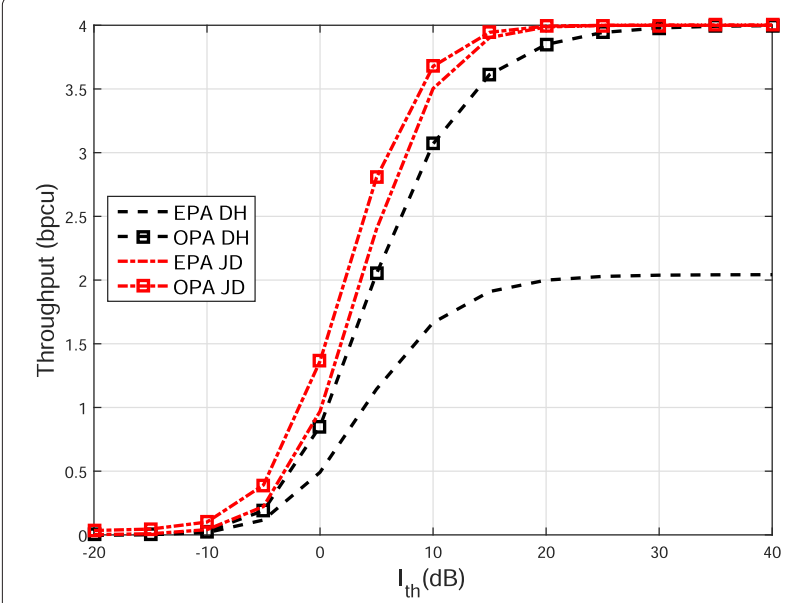

Fig. 10 Throughput for the different FD schemes as a function of the primary interference threshold $I_{\text {th }}$, with $\mathcal{R}=4$ bpcu, $P_{p}=10 \mathrm{~dB}$, and $\delta=-40 \mathrm{~dB}$

$Z_{k l}$ as $f_{Z_{k l}}\left(z_{k l}\right)=\lambda_{k l} \lambda_{k p} /\left(\lambda_{k l}+\lambda_{k p} z_{k l}\right)^{2}$, while the CDF $F_{Z_{k l}}\left(z_{k l}\right)=1-\lambda_{k l} /\left(\lambda_{k l}+\lambda_{k p} z_{k l}\right)$.

\section{Appendix 2: PDF and CDF of W}

We recall that $W=Z_{s s}+Z_{s r}$ and $f_{Z_{k l}}\left(z_{k l}\right)=\frac{\lambda_{k l} \lambda_{k p}}{\left(\lambda_{k l}+\lambda_{k p} z_{k l}\right)^{2}}$ [29]. Thus, the PDF $f_{W}(w)$ is attained, with the help of ([43] Eq.(2.154), (2.155)), as

$$
\begin{aligned}
f_{W}(w)= & \int_{0}^{w} f_{Z_{s s}}\left(z_{s s}\right) f_{Z_{r s}}\left(w-z_{s s}\right) \mathrm{d} z_{s s} \\
= & \lambda_{r s} \lambda_{s s} \mu_{s} \frac{w\left(\lambda_{r s} \lambda_{s p} \mu_{s}+\lambda_{r p} \lambda_{s s} \mu_{s}+\lambda_{r s} \lambda_{s s} w\right)}{\left(\lambda_{r p} \mu_{s}+\lambda_{r s} w\right)\left(\lambda_{s p} \mu_{s}+\lambda_{s r} w\right)\left(\lambda_{r s} \lambda_{s p} \mu_{s}+\lambda_{r p} \lambda_{s s} \mu_{s}+\lambda_{r s} \lambda_{s s} w\right)^{3}} \\
& \times \frac{\left(\mu_{s}\left(\lambda_{r s}^{2} \lambda_{s p}^{2}+\lambda_{r p}^{2} \lambda_{s s}^{2}\right)+\lambda_{r s} \lambda_{s s} w\left(\lambda_{r s} \lambda_{s p}+\lambda_{r p} \lambda_{s s}\right)\right)}{\left(\lambda_{r p} \mu_{s}+\lambda_{r s} w\right)\left(\lambda_{s p} \mu_{s}+\lambda_{s r} w\right)\left(\lambda_{r s} \lambda_{s p} \mu_{s}+\lambda_{r p} \lambda_{s s} \mu_{s}+\lambda_{r s} \lambda_{s s} w\right)^{3}} \\
& +\frac{2 \lambda_{r p} \lambda_{s p}\left(\lambda_{r s} \lambda_{s s} \mu_{s}\right)^{2}}{\left(\lambda_{r s} \lambda_{s p} \mu_{s}+\lambda_{r p} \lambda_{s s} \mu_{s}+\lambda_{r s} \lambda_{s s} w\right)^{3}} \ln \left(\frac{\left(\lambda_{r p} \mu_{s}+\lambda_{r s} w\right)\left(\lambda_{s p} \mu_{s}+\lambda_{s s} w\right)}{\lambda_{r p} \lambda_{s p} \mu_{s}^{2}}\right),
\end{aligned}
$$

while the CDF $F_{W}(w)$ is obtained by integrating (49) from 0 to $\frac{\epsilon}{\mu_{s}}$, which results in (11).

\section{Competing interests}

The authors declare that they have no competing interests.

\section{Acknowledgements}

This work was partially supported by the University of Oulu Graduate School, Infotech Oulu Graduate School, Academy of Finland, Araucaria Foundation (Brazil), CAPES (Brazil), and CNPq (Brazil).

\section{Author details}

${ }_{1}^{1}$ Federal University of Technology_Paraná (UTFPR), 80230-901 Curitiba, Brazil. ${ }^{2}$ Centre for Wireless Communications (CWC), University of Oulu, Oulu, Finland. ${ }^{3}$ Federal University of Ceará (UFC), Fortaleza, Brazil. ${ }^{4}$ Federal University of Paraná (UFPR), Curitiba, Brazil.

Received: 30 November 2014 Accepted: 23 May 2015

Published online: 12 June 2015

\section{References}

1. A Goldsmith, SA Jafar, I Maric, S Srinivasa, Breaking spectrum gridlock with cognitive radios: an information theoretic perspective. Proc. IEEE. 97(5), 894-914 (2009)

2. S Srinivasa, SA Jafar, The throughput potential of cognitive radio: a theoretical perspective. IEEE Commun. Mag. 45(5), 73-79 (2007)

3. A Ghasemi, ES Sousa, Fundamental limits of spectrum-sharing in fading environments. IEEE Trans. Wireless Commun. 6(2), 649-658 (2007)

4. L Lu, X Zhou, $U$ Onunkwo, G Li, Ten years of research in spectrum sensing and sharing in cognitive radio. EURASIP J. Wirel. Commun. Netw. 2012(1), 28 (2012)

5. JN Laneman, DNC Tse, GW Wornell, Cooperative diversity in wireless networks: efficient protocols and outage behavior. IEEE Trans. Inf. Theory. 50(12), 3062-3080 (2004)

6. A Nosratinia, TE Hunter, A Hedayat, Cooperative communication in wireless networks. IEEE Commun. Mag. 42(10), 74-80 (2004)

7. H Alves, R Souza, G Fraidenraich, M Pellenz, Throughput performance of parallel and repetition coding in incremental decode-and-forward relaying. Wirel. Netw. 18(8), 881-892 (2012)

8. G Kramer, M Gastpar, P Gupta, Cooperative strategies and capacity theorems for relay networks. IEEE Trans. Inf. Theory. 51(9), 3037-3063 (2005)

9. TKwon, S Lim, S Choi, D Hong, Optimal duplex mode for df relay in terms of the outage probability. IEEE Trans. Veh. Technol. 59(7), 3628-3634 (2010)

10. M Duarte, C Dick, A Sabharwal, Experiment-driven characterization of full-duplex wireless systems. IEEE Trans. Wireless Commun. 11(12), 4296-4307 (2012)

11. TRiihonen, S Werner, R Wichman, Mitigation of loopback self-interference in full-duplex mimo relays. IEEE Trans. Signal Process. 59(12), 5983-5993 (2011)

12. TRiihonen, S Werner, R Wichman, Hybrid full-duplex/half-duplex relaying with transmit power adaptation. IEEE Trans. Wireless Commun. 10(9), 3074-3085 (2011)

13. TRiihonen, $\mathrm{S}$ Werner, R Wichman, Optimized gain control for single-frequency relaying with loop interference. IEEE Trans. Wireless Commun. 8(6), 2801-2806 (2009)

14. H Alves, G Fraidenraich, RD Souza, M Bennis, M Latva-aho, in Wireless Communications and Networking Conference (WCNC), 2012 IEEE. Performance analysis of full duplex and selective and incremental half duplex relaying schemes (Paris, France, 2012), pp. 771-775. doi:10.1109/WCNC.2012.6214475

15. H Alves, RD Souza, G Fraidenraich, Outage, throughput and energy efficiency analysis of some half and full duplex cooperative relaying schemes. Trans. Emerg. Telecommun. Technol. 25(11), 1114-1125 (2014)

16. Y Zhu, Y Xin, P-Y Kam, Outage probability of rician fading relay channels. IEEE Trans. Veh. Technol. 57(4), 2648-2652 (2008)

17. H Alves, D Benevides da Costa, RD Souza, M Latva-aho, Performance of block-markov full duplex relaying with self interference in nakagami-m fading. IEEE Wireless Commun. Lett. 2(3), 311-314 (2013)

18. TQ Duong, PL Yeoh, VNQ Bao, M Elkashlan, N Yang, Cognitive relay networks with multiple primary transceivers under spectrum-sharing. IEEE Signal Process. Lett. 19(11), 741-744 (2012)

19. TQ Duong, VNQ Bao, H Tran, GC Alexandropoulos, H-J Zepernick, Effect of primary network on performance of spectrum sharing AF relaying. Electron. Lett. 48(1), 25-27 (2012)

20. TQ Duong, D Benevides da Costa, M Elkashlan, VNQ Bao, Cognitive amplify-and-forward relay networks over nakagami-m fading. IEEE Trans. Veh. Technol. 61(5), 2368-2374 (2012)

21. TQ Duong, D Benevides da Costa, TA Tsiftsis, C Zhong, A Nallanathan, Outage and diversity of cognitive relaying systems under spectrum sharing environments in nakagami-m fading. IEEE Commun. Lett. 16(12), 2075-2078 (2012)

22. W Xu, J Zhang, P Zhang, C Tellambura, Outage probability of decode-and-forward cognitive relay in presence of primary user's interference. IEEE Commun. Lett. 16(8), 1252-1255 (2012)

23. S Mafra, R Souza, J Rebelatto, E Fernandez, H Alves, Cooperative overlay secondary transmissions exploiting primary retransmissions. EURASIP J. Wirel. Commun. Netw. 2013(1), 196 (2013)

24. VNQ Bao, TQ Duong, D Benevides da Costa, GC Alexandropoulos, A Nallanathan, Cognitive amplify-and-forward relaying with best relay 
selection in non-identical rayleigh fading. IEEE Commun. Lett. 17(3), 475-478 (2013)

25. J Si, Z Li, H Huang, J Chen, R Gao, Capacity analysis of cognitive relay networks with the PU's interference. IEEE Commun. Lett. 16(12), 2020-2023 (2012)

26. X Jia, M Zhou, X Dang, L Yang, H Zhu, Adaptive power allocation and outage performance of cognitive best relay cooperation systems with multiple primary transceiver pairs and direct path between cognitive source and destination. EURASIP J. Wirel. Commun. Netw. 2014(1), 122 (2014)

27. F Zhao, X Sun, H Chen, R Bie, Outage performance of relay-assisted primary and secondary transmissions in cognitive relay networks. EURASIP J. Wirel. Commun. Netw. 2014(1), 60 (2014)

28. EE Benitez Olivo, DP Moya Osorio, DB Costa da, JC Silveira Santos Filho, Outage performance of spectrally efficient schemes for multiuser cognitive relaying networks with underlay spectrum sharing. IEEE Trans. Wireless Commun. 13(12), 6629-6642 (2014). doi:10.1109/TWC.2014.2360204

29. H Kim, S Lim, H Wang, D Hong, Optimal power allocation and outage analysis for cognitive full duplex relay systems. IEEE Trans. Wireless Commun. 11(10), 3754-3765 (2012)

30. M Khafagy, A Ismail, M-S Alouini, S Aissa, On the outage performance of full-duplex selective decode-and-forward relaying. IEEE Commun. Lett. 17(6), 1180-1183 (2013)

31. MG Khafagy, A Tammam, M Alouini, S Aissa, Efficient cooperative protocols for full-duplex relaying over nakagami-m fading channels. IEEE Trans. Wireless Commun. (2015). doi:10.1109/TWC.2015.2406712

32. S Mafra, H Alves, RD Souza, D Benevides da Costa, E Fernandez, M Latva-aho, in 31st Brazilian Telecommunications Symposium - SBrT'13. On the performance of cognitive full-duplex relaying systems under spectrum sharing constraints (Fortaleza, Brazil, 2013)

33. T Cover, AE Gamal, Capacity theorems for the relay channel. IEEE Trans. Inf. Theory. 25(5), 572-584 (1979)

34. A Carleial, Multiple-access channels with different generalized feedback signals. IEEE Trans. Inf. Theory. 28(6), 841-850 (1982)

35. C-M Zeng, F Kuhlmann, A Buzo, Achievability proof of some multiuser channel coding theorems using backward decoding. IEEE Trans. Inf. Theory. 35(6), 1160-1165 (1989)

36. A Jovicic, P Viswanath, Cognitive radio: an information-theoretic perspective. IEEE Trans. Inf. Theory. 55(9), 3945-3958 (2009)

37. E Pei, S Wang, Z Zhang, Capacity and optimal power allocation for spectrum-sharing with primary transmission consideration in fading channels. IEEE Commun. Lett. 15(4), 389-391 (2011)

38. X Gong, SA Vorobyov, C Tellambura, Optimal bandwidth and power allocation for sum ergodic capacity under fading channels in cognitive radio networks. IEEE Trans. Signal Process. 59(4), 1814-1826 (2011)

39. A Punchihewa, VK Bhargava, C Despins, Capacity and power allocation for cognitive mac with imperfect channel estimation. IEEE Trans. Wireless Commun. 10(12), 4001-4007 (2011)

40. G Caire, D Tuninetti, The throughput of hybrid-arq protocols for the gaussian collision channel. IEEE Trans. Inf. Theory. 47(5), 1971-1988 (2001)

41. VNQ Bao, DH Bac, in ICT Convergence (ICTC), 2011 International Conference On. A unified framework for performance analysis of df cognitive relay networks under interference constraints (Seoul, South Korea, 2011), pp. 537-542. doi:10.1109/ICTC.2011.6082656

42. A Papoulis, SU Pillai, Probability, Random Variables, and Stochastic Processes. (Tata McGraw-Hill, New York, 2002)

43. IS Gradshteyn, IM Ryzhik, Table of Integrals, Series, and Products, Fifth Edition. (Academic Press, New-York, 2007)

\section{Submit your manuscript to a SpringerOpen ${ }^{\odot}$ journal and benefit from:}

- Convenient online submission

Rigorous peer review

- Immediate publication on acceptance

- Open access: articles freely available online

- High visibility within the field

- Retaining the copyright to your article

Submit your next manuscript at $\boldsymbol{\triangleright}$ springeropen.com 